XII.

\title{
Ueber die Wirkung des Cholin und des Neurin.
}

Ein Beitrag zur Kenntniss der Gefässgifte.

Von

\author{
J. Pal (Wien). \\ (Jierzu Tafel V.)
}

\section{Einleitung.}

Wir sind gewöhnt, unter den Gefässmitteln nach ihrer Hauptwirkung vasoconstrictorisch wirkende von vasodilatatorisch wirkenden zu unterscheiden. Diese Unterscheidung ist aber nach dem gegenwärtigen Stande unseres Wissens hinfällig, da bereits von eminent vasoconstrictorisch bezeichneten Agentien festgestellt ist, dass sie in einzelnen Gefïssgebieten direct dilatatorisch wirken; so wirkt das Adrenin auf die Kranzarterien (Langendorff), das Pituitrin infund. auf die Nierengefässe dilatatoriseh ${ }^{\mathbf{l}}$ ). Man könnte sonach eigentlich nur von pressorisch und von depressorisch wirkenden Gefässgiften sprechen. Aber auch diese Trennung, wenngleich sie binsichtlich der sinnfälligen Hauptwirkung zu Recht besteht, entspricht nicht absolut den Thatsachen. Selbst das drucksteigernde Adrenin wirkt, wie aus den Versuchen von Dale hervorgeht, nicht ausschliesslich pressorisch. Schaltet man nämlich nach Dale die Ansprechbarkeit der Vasoconstrictoren durch Ergotoxin aus, so tritt nach Adrenin eine depressorische Wirkung ein. Auch im Blutdruckseffect des Hypophysenextractes haben wir es typisch mit einer Depression zu thun, die dann in eine pressorische Wirkung übergeht. Bei der zweiten Injection tritt sogar in der Regel - nicht immer, wie ich gezeigt habel - - die pressorische Wirkung zurück. Die Substanz, die wir im Extract des hinteren Lappens der Hypophyse vor uns haben (Pituitrin P. D. Co.) ist zwar eine chemisch nicht definirte, doch derart gleichmässig in der Wirkung, dass wir mit einer einheitlichen Substanz rechnen müssen. C. A. Schäfer hat allerdings angegeben, dass er aus seinem Extract zwei Körper trennen konnte, von denen der eine depressorisch, der andere pressorisch wirke. Aus dem Pituitrin der Firma Parke, Davis \& Co. ist mir dies nicht gelungen, auch haben die über meinen Wunsch in Detroit ausgeführten

1) Vergl. Pal: Sitzungsber. der k. k. Ges. der Aerzte in Wien yom 4. Dec. 1908. Wiener med. Wochenschr. Jan. 1909. 
Versuche zur Gewinnung der beiden Substanzen kein positives Resultat ergeben.

Was sich bei diesen eminent pressorisch wirkenden Substanzen nachweison lässt, beobachten wir noch ausgezeichneter bei manchen anderen Gefässgiften, und namentlich beim Cholin und dem Neurin. Wenn man mit diesen beiden Giften experimentirt, kann man unter Umständen zu ganz widersprechenden Ergebnissen gelangen. Es kann sich dies nur dadurch erklären, dass diese Gifte depressorische und pressorische Wirkung haben und unter gewissen Bedingungen die eine, unter Umständen die andere dominirt. Bei den Versuchen mit dem Cholin und dem Neurin im Besonderen habe ich erfahren, dass einerseits zunächst nicht näher feststellbare, ganz individuelle Reactionsfähigkeit des Thieres und andererseits die Versuchsbedingungen und unter diesen namentlich die Art und Dauer der Narkose auf den Ablauf der Gefässreaction bestimmend wirken.

Wir haben ein anderes Bild der Gefässwirkung nach der Anwendung von Curare oder in der Aethernarkose, wie bei Thieren, die durch Ausschaltung des Centralnervensystems immobilisirt werden.

Die aus solchen Versuchen sich ergebende Thatsache, dass Gefüssgifte unter Umständen in ihrer Wirkung völlig umschlagen, d. h. geradezu conträr wirken können, ist eine nicht allein rein toxicologisch, sondern auch klinisch wichtige Angelegenheit.

Dass eine solche Abänderung der Wirkung unter dem Einfluss gewisser, dem Organismus vorher einverleibter Substanzen eintritt, ist nach den Versuchen von Langloy, Dale u. A. keine Neuhoit, dass aber die gangbaren Versuchsanordnungen neben der rein individuellen Ansprechbarkeit des Thieres so grosse Differenzen bedingen, ist bisher, wie gerade die Erfahrung beim Cholin aus der jüngsten Zeit demonstrirt, nicht berücksichtigt worden.

\section{Ueber die Cholinwirkung.}

In den letzten Jahren ist vielfach die Aufmerksamkeit auf das Cholin gelenkt und seinem Vorkommen unter physiologischen und pathologisehen Bedingungen besondere Bedeutung beigelegt worden. Man hat aus einer Reihe von Organen, namentlich aus Blutdrüsen, Substanzen dargestellt, die als Cholin angesprochen wurden. Man hat geglaubt, bei gewissen Krankheiten, wie Epilepsie, progressiver Paralyse, im Blute und in der Cerebrospinalflüssigkeit Cholin nachgewiesen zu haben. Da dem Cholin krampferregende Eigenschaften zukommen, war man der Meinung, die Quelle der Krampfzustände gefunden zu haben (Donath).

Ein weiterer und wichtiger Grund, das Vorkommen des Cholin zu verfolgen, ergab sich aus dem Umstande, dass das Cholin als eine rein depressorisch wirkende Substanz und als directer Antagonist des Nebennierenproductes (des Adrenin) erklärt wurde (Johmann, Desgrez und Chevalier u. $\Lambda$.).

Lis soll hier nicht untersucht werden, inwiefern diese Bewerthung des Cholins im Thierkörper überhaupt eine Berechtigung hat, aber angesichts der supponirten Wichtigkeit des Cholins war es doch merk- 
würdig, dass die Frage nach seiner eigentlichen Wirkung so verschieden beantwortet wurde.

Der wichtigste Widerspruch ergab sich in der Blutdruckwirkung, die von den Einen als vorwiegend pressorische, von den Anderen als rein depressorische bezeichnet wird. Es geht dies so weit, dass einzelne Untersucher in dem Umstande, dass ihre Substanz eine depressorische Wirkung verursachte, einen Beweis sehen, dass sie Cholin in Händen haben.

Bei der Differenz in den Resultaten musste man zunächst daran denken, dass die versehiedenen Untersucher nicht die gleiche Substanz in Händen hatten. Diesen Schluss zog Modrakowski1). Er fand, dass das Cholin und die verschiedenen Cholinpräparate sehr zersetzlich sind, was an dem bald auftretenden Trimethylamingeruch kenntlich ist. Auf diese Weise handelte es sich bei den Versuchen nicht mehr um die Cholinwirkung, sondern auch um die Mitwirkung seiner Zersetzungsproducte, die unter Anderem auch muscarinartige Wirkung haben. Das Cholin ist bekanntlich dem Muscarin nahe verwandt.

Die verschiedenen Intersucher benutzten theils selbst bereitete, theils von Merck, Kahlbaum a. A. hergestellte Präparate.

Eine gewisse Schwierigkeit in der Klärung dieser Angelegenheit hat sich neuerdings dadureh ergeben, dass es sich gezeigt hat, dass es eine ganze Reihe von Cholinkörpern giebt.

In den Arbeiten von Modrakowski sind die wichtigsten literarischen Angaben schon enthalten. Es ist also eigentlich unnöthig, sie hier nochmals zu reproduciren. Einige muss ich aber doch rerzcichnen, weil sie für meine Ausführungen von Belang sind.

$\Lambda$ s die wesentliehen Merkmale der Cholinwirkung werden die Wirkung auf das Herz und die Arterien, bezw. auf den Blutdruck, ferner auf die Athmung und die secernirenden Drüsen angesehen.

Die Frage nach der Giftigkeit des Cholins wird sehr verschiedenartig beantwortet. Viele leugnen eigentlich seine T'oxicitat (Boehm u. A.), andere sprechen von tödtlichen Dosen. So giebt Corvello 0,3, II. C. Wo od 0,5 pro Kilogramm als tüdtliche Dosis an, während L,ohmann 0,03 für $1,5 \mathrm{~kg}$ annimmt.

Eine ganze Reihe von Autoren berichtet über rein depressorische Wirkung des Cholins, so Mott und Halliburton, Lohmann, Desgrez und Chevalier, v. Fürth und Schwarz, Gautrelet u. A.

Mott und Halliburton fanden bei Hunden und Katzen in der A. C. E.-Narkose oder auch nach Morphin bei Anwendung von 0,001 bis 0,014 Cholin pro Kilogramm Körpergewicht Blutdrucksenkung. Diese soll zu Beginn eardial, im Uebrigen von einer Krweiterung der splanchnischen Gefässe abhängig sein. Die Respiration wird nicht beeinflusst. Erst nach Atropin wirkt Cholin nur drucksteigernd. Mott und Halliburton bezeichnen dies für das Cholin als charakteristisch. Den Wegfall der Depression nach Atropin haben übrigens fast alle Untersucher

1) Arch. f. Phys. 1908. Bd. 124. S. 601. 
der Cholinwirkung bestätigt (Schäfer und Vincent, Abderhalden und Fr. Müller u. v. A.).

Allein schon Gaehtgens, von dem die crste Untersuchung der Cholinwirkung stammt, hat gefunden, dass Cholin erst ein Ansteigen und dann ein Fallen des Blutdrucks bewirke. Grosse Dosen tödten durch Athmungslähmung.

Böhm, der, abgesehen von Salivation bei Katzen, die er am empfindlichsten fand, niemals deutliche Vergiftungserscheinungen sah, beobachtete nach intravenöser Injection von 0,01 bis 0,02 Cholin rasch vorübergehende geringfügige Drucksteigerung. Blutdrucksteigerung, der eine vorübergehende Drucksenkung vorausging, haben Asher und Wood beschrieben, gelegentlich haben sie auch Osborne und Vincent beobachtet.

Formanek ${ }^{1}$ ), der morphinisirten und curaresirten Hunden 0,04 bis 0,06 Cholin injicirte, fand anfängliche vorübergehende Blutdrucksenkung, dann erheblicheren Anstieg des Blutdrucks unter Verlangsamung der Herzaction, dabei Zunahme der Amplituden der einzelnen Herzsehläge. Nach Durchschneidung der Vagi oder nach vorausgegangener Atropinisirung trat keine Pulsverlangsamung auf, auch blieb die anfängliche Drucksenkung dann oft aus. Bei aufeinanderfolgenden Injectionen von Cholin zeigten sich die späteren weniger wirksam als die erste. Die Blutdrucksenkung ist nach Formanek wahrscheinlich, die Pulsbeschleunigung sicher eine primäre Wirkung auf das Herz. Die Pulsverlangsamung beruht auf Reizung der centralen Vagusenden, während die Drucksteigerung durch Reizung des peripheren vasoconstrictorischen Apparates im Splanchnicusgebiet bedingt ist. Modrakowski (1908) hat mit dem friseh hergestellten Kahlbaum'schen Präparat, sowie mit seinem selbst gewonnenen nur blutdrucksteigernde Wirkung ersielt, insofern das Präparat nicht der Zersetzung anheimgefallen war.

Seit dem Erscheinen der Arbeit Modrakowski's liegen noch einige Veröffentlichungen vor, die die Cholinwirkung in schr widersprechender Weise darstellen. Da sind vor Allem die Arbeiten von Desgre\% und Chevalier ${ }^{2}$, die dem Cholin eine ausgesprochene depressorische Wirkung zusehrciben. J. Gautrelet'3), der zu dem gleichen Resultat gelangte, will sogar einen cholinogenen Apparat von einem chromaffinen unterscheiden.

Busquet und Pachon ${ }^{4}$ berichten, dass das Cholin in geringen Dosen (bis $2 \mathrm{mg}$ pro $1 \mathrm{~kg}$ ) vorübergehende Drucksenkung erzeuge, während bei grösseren Gaben die pressorische Wirkung anhalte, und zwar länger als die depressorische. Ganz conform lauten die Angaben von J. Parisot ${ }^{5}$ ), der bei Kaninchen das Cholin (salzsaures Cholin, Poulenc) in Gaben von 8 bis $10 \mathrm{mg}$ pro Kilo nach kurzer Senkung ausgiebige Drucksteigerung

1) Areh. internat. de pharmacodyn. et de thérapie. 1902. Vol. X. p. 177.

2) Compt. rend. 1908. Vol. 146. p. 89.

3) Bull. de la soc. de biol. T'. 65. p. 173 ध. 448. -- Compt. rend. 1909. Vol. 148. p. 995.

4) Bull. de la soc. de biol. 1909. T. 67. p. 218.

5) Ibid. T. 6i. p. 749 . 
verursachen sah. Busquet und Pachon') zeigten übrigens, dass sich das Cholin und Adrenalin in ihrer Wirkung summiren und nicht aufheben.

In No. 1 des "Centralblatts für Physiologie",, Bd. XXIV ${ }^{2}$ ) habe ich eine kurze Mittheilung über die Cholinwirkung bereits veröffentlicht, deren ausführliche Darstellung die vorliegende Arbeit giebt.

Bald nach meiner Mittheilung ist eine Arbeit von Emil $\Lambda$ bderhalden und Franz Müller ${ }^{3}$ ) erschienen, die als die eigentliche Wirkung des Cholins die depressorische Blutdruckwirkung bezeichnen. Sie führen immerhin Beobachtungen an, in welchen — es handelt sich um Thiere mit durchtrennter Medulla - Drucksteigerung eintrat. Später folgte noch eine weitere Mitheilung von Franz Müller, die sich mit der Analyse der Cholinwirkung ausführlich beschäftigt. Auch in dieser wird die primäre Erweiterung der Gefässe betont und nur die Vasoconstriction und Drucksteigerung nach Atropin zugegeben, die als Folge der Lähmung der dilatatorischen Elemente anzuschen ist ${ }^{4}$ ).

\section{Eigene Untersuchungen.}

Mit Untersuchungen über die Wirkung des Cholins habe ich mich bereits 1907/8 beschäftigt, und zwar gleichzeitig mit den bereits verölfentlichten Studien über die Wirkung des Hypophysenextractes ${ }^{5}$ ). Das reine Cholin, das ich damals durch die Höchster Farbwerke erhielt, erwies sich als nicht haltbar, zeigte eminent toxische und muscarinartige Wirkung, erweiterte das überlebende Gefäss des Rindes und die Pupille des ausgeschnittenen Froschbulbus.

Im Jahre 1909 gelangte ich durch die Freundlichkeit der Höchster Farbwerke in den Besitz eines nach der Methode von Wurtz synthetisch hergestellten salzsauren Cholins. Angesichts der Angaben über die Zersetzlichkeit des Präparates, sowie auf Grund meiner eben crwähnten Erfahrungen mit dem reinen Cholin, habe ich auf die Haltbarkeit des Präparates mein besonderes Augenmerk gerichtet. Ich hatte auch Grund, die Zersetzlichkeit in Erwägung zu ziehon. Allein die Stichproben, die ich mit dem von den Höchster Farbwerken crhaltenen Präparat ausführte, zeigten, dass es als vollkommen haltbar bezeichnet werden musste. Weder das Offenstehenlassen, noch das Auftauchen von Schimmelpilzen, Durchleiten von $\mathrm{O}$, Vermengung mit verdünnter $\mathrm{I}_{2} \mathrm{O}_{2}-\mathrm{I}$,ösung, Belichtung usw. hatten einen bestimmten Einfluss auf das Ergebniss meiner

1) Ibid. T. 67. p. 277 .

2) Erschienen am 2. April 1910.

3) Zeitschr. f. physiol. Chemie. 1910. Bd. 65. S. 420. - Med. Klinik. 1910. No. 22. - Arch. f. Physiol. 1910. Bd. 134.

4) Der Vollständigkeit halber erwähne ich hier, dass inzwischen am internationalen Physiologencongress, anschliessend an cine Mittheilung von Gau tr c let (28. Sept. 1910) eine Discussion bezüglich der Cholinwirkung stattgefunden hat, in der ich meine hier vertretenen Anschauungen zum Theil vorgebracht habe.

5) Sitzung der k. k. Gesellschaft der Aerzte in Wien vom 4. December 1908, ferner Wiener med. Wochenschr. Januar 1909. 
Expcrimente. Gewisse Unterschiede in der Wirkung mussten also anderweitig ihre Erklärung finden.

Meine Ausführungen bezichen sich auf 50 Versuche, die ich mit dem synthetischen salzsauren Cholin (Höchst) angestellt habe. Sie betreffen 4 Hunde, 45 Katzen und 1 Kaninchen.

Die kymographisehen Versuche sind zum grössten Theil (41) im Institute für allgemeine und experimentelle Pathologic der Wicner Universität (Hofrat Paltauf), ein kleiner Theil in meinem Experimentallaboratorium ausgeführt. Ich beziehe mich hier, wo dies nicht ausdrücklich vermerkt ist, auf Beobachtungen an Katzen. An dieser Thiorspecies habe ich meine Untersuchungen schon aus dem Grunde fortgeführt, weil sie in der Literatur als für Cholin besonders empfindlich erklärt wurden.

Injicirt habe ich Dosen von 0,005-0,2, auf das Körpergewicht bezogen, 0,0015-0,12 pro Kilo bei den Katzen, 0,005-0,015 bei Hunden.

Das Cholin wirkt ganz verschieden, jo nachdem es subcutan oder intravenös applicirt wird.

Ein Kaninchen ron $2 \mathrm{~kg}$ Gewicht hat wiederholte Gaben von 0,1 bis 0,2 (subcutan) gut vertragen. Die Harnmenge war einmal etwas vermehrt. Bei der grösseren Dose war das Thier einige Stunden nachher etwas hinfüllig. Glykosurie trat nicht auf.

Bei einer Katze $(2 \mathrm{~kg})$ beobachtete ich nach ciner subcutanen Injection von 0,2 eine Viertelstunde Unruhe, Andeutung von Muskelzittern, Speichelfluss, auch Thränen erschienen. Diese Symptome liefen bald ab, und nach einer halben Stunde war das Thicr in seiner früheren Verfassung.

Ich habe die Wirkung der subcutanon Injection des Cholins auch am curaresirten Thiere (Katze $5 \mathrm{~kg}$ ) geprït und auch an einer Katze $(2,7 \mathrm{~kg})$ nach Durchtrennung der Medulla oblongata. In beiden Fällen traten - im crsteren nach 16 Minuten, im zwciten schon nach 3 Minuten - Zuckungen auf. Beim curaresirten Thiere stellte sich nach 16 Minuten eine kurz vorübergehende Drucksteigerung von 84 auf $124 \mathrm{~mm}$ ein, bei der anderen Katze nach 11 Minuten von 76 auf $100 \mathrm{~mm}$.

Die Hunde wurden in Chloroformnarkose, die Katzen in Acthernarkose tracheotomirt, dann euraresirt oder die Oblongata durchtrennt. In einigen Fällen wurde das Rückenmark zerstört. In der Regcl wurden von vornhercin die Halsvagi durchtrennt, der Blutdruck aus der Carotis registrirt. Die intravenösen Injectionen erfolgten in die Vena jugularis externa.

Meine Blutdruckversuche ergaben, dass das Cholin bei intravenöser Einbringung in der Regel cine kurze Depression erzeugt, dic von einer oft beträchllichen Drucksteigerung gefolgt wird.

Es zeigte sich bald, dass das Ergebniss von ciner Reihe von Bedingungen abhängig ist, vor Allem von ganz individucllen des Thieres, dann von der Grösse der Einzelgabe und schliesslich von Versuchsbedingungen, unter denen sich die Narkose, der Zustand des Thieres als die wichtigsten erwiesen. Dass individuelle Momente boi der Cholinwirkung ausschlaggebend sind, zeigt ein an 2 Hunden ausgeführter Parallelversuch. Obwohl dieselbe Lösung unter ganz genau eingehaltenen 
gleichen Bedingungen zur Anwendung gelangte, hatte das eine Thier bei durchtrenten Halsvagis - starke und anhaltende Vagusreizungserscheinungen, während diese beim anderen Thiere kaum angedeutet waren.

Bezüglich der Herzwirkung fand ich, dass sie auch bei der Katze eine sehr wechselnde ist. In den meisten Fällen tritt bei mittleren Dosen Pulsverlangsamung auf, weniger häufig kommt es zu ausgeprägten Vaguspulsen - trotzdem aber, namentlich bei Hunden, zur Drucksteigerung. Die Vagusreizung ist nach gewissen Curaresorten sehr ausgeprägt. Bei einer Curareart kam es (bei Katzen) immer zu muscarinartiger Wirkung. In 3 Versuchen habe ich mit dem Rothberger'schen Onkometer das Herzvolumen registrirt. Aus diesen Versuchen ist wohl hervorgegangen, dass die Vaguserscheinungen directe Herzwirkungen sind und durch $\Lambda$ tropin beseitigt werden. Ueber die Genese der Depression geben sie im Uebrigen keinen Aufschluss. Es liegen von anderer Seite Versuche vor (Abderhalden und Müller), die für periphere Dilatation sprechen. Diese Versuche habe ich nicht wiederholt.

Trotz genügender Atropingaben fällt die Depression nicht immer ganz weg und es kommt mitunter nur zu ganz geringer Drucksteigerung (vergl. Vers. 25. 11. 1909), so dass ich an dem zum Theil peripheren Ursprung der Depression nicht zweifeln kann.

Der Eintritt der Depression und deren Verlauf ist, sowie überhaupt die Gestaltung der Blutdruckwirkung, von der Narkose abhängig.

Nach starken und anhaltenden Aethernarkosen (vergl. Versuch vom 17. 5. 1910), sowie nach Curare tritt bei Katzen die depressorische Wirkung stark hervor und die pressorische kommt mitunter wenig oder auch überhaupt nicht zu Stande. Dorh muss ich hinzufügen, dass es hinsichtlich des Curare auf dessen Qualität ankommt. In letzter Zeit habe ich Curaresorten probirt, bei deren Anwendung ein pressorischer Effect ohne Atropin überhaupt nicht erziclt werden konnte ${ }^{1}$ ). In diesem Falle war auch meist starke Vagusreizung da.

Mitunter gelingt es, durch entsprechendes Zuwarten nach der Curaregabe einen pressorischen Effect zu erzielen.

Wirkt das Cholin nicht pressorisch, so wirken auch nach grossen Dosen Cholin Adreninpräparate in kleinen Mengen, wenn auch nicht immer wie beim normalen Thiere, ganz deutlich drucksteigernd.

Bezüglich des Einflusses der Einzelgaben habe ich gefunden, dass die kleinste eben wirksame Dosis, die bei den verschiedenen Thicren sehr verschieden hoch liegt, ceteris paribus vorwiegend depressorisch wirkt und der pressorische Effect nur gering ist. Bei Wiederholung der gleichen Gabe tritt meist cine Abschwächung ein, nach einer Reihe von Injectionen fehlt oft jede Wirkung. Die schönsten pressorischen Effecte mit in der Regel nur geringen initialen Depressionen sind nach relativ kleinen Dosen bei gut erhaltenen Thieren nach Durchtrennung der Medulla oblongata $z u$ beobachten (s. Beispiele in den Versuchsprotokollen vom 11. 1. $1910,26.1 .1910$ etc.).

Injicirt man bei einem solchen Thiere, nachdem die pressorische

1) Vergl. Modrakowski, Arch. f. Phys. Bd. 233. 
Reaction festgestellt wurde, Curare, so bleibt nach einer daraufhin folgenden Cholingabe der pressorische Effect aus, oder er wird auf ein Minimum reducirt (vergl. 17. 2. 1910), selbst wenn die Dosis erhöht wurde.

Die Drucksteigerung erfolgt bei durchtrennter Oblongata - also bei geringer Narkosewirkung - meist sehr rasch. Die Curve wird steil, ähnlich wie nach Adrenimpräparaten. Die Drucksteigerung tritt auch nach Durchtrennung der Splanchnici und Entfernung des Rückenmarks cin und ist sonach, wie schon Formanek festgestellt hat, jedenfalls durch periphere Wirkung bedingt (Versuch 26. 1. 1910, 16. 2. 1910 u. A.)

Die Annahme von Fr. Müller, dass die Drucksteigerung durch Krampfreizung hervorgerufen werde, muss ich auf Grund von zahlreichen Beobachtungen, in denen jede Muskelbewegung fehlte, als unhaltbar bezeichnen (vergl. die diesbezüglichen Hinweise in den Versuchspratokollen).

An curaresirten Thieren ist ferner Folgendes zu beobachten: Eine gewisse Dosis des Cholins, dessen Höhe von dem betreffenden Curarepräparat abhängt, sowie von seiner Dosirung, hebt die Curarewirkung sofort auf; gewöhnlich genügen 0,02. Bei grösseren Dosen kommt es vor, dass das Thier schon während der Injection Beissbewegungen macht (Vers. vom 25. 11. 1909). Die spontane Athmung, die nun einsetzt, ist eine stark beschleunigte; sie ist von Muskelzittern, oft auch von klonischen Krämpfen begleitet, sowie von starkem Speichel- und Thränenfluss.

Die Aufhebung der Curarewirkung tritt hier noch rascher ein als beim Physostigmin, dessen diesbezügliche Wirkung ich 1900 entdeckt habe ${ }^{1}$ ).

Die physostigminähnliche Wirkung des Cholins heben auch E. Abderhalden und Fr. Müller hervor. Allein der Effect ist beim Cholin nicht nachhaltig. Das Thier athmet wohl einige Zeit spontan fort. In der Regel versiegt aber die Athmung nach einigen Minuten und das Thier muss wieder künstlich geathmet werden. Wird die Cholingabe wiederholt, so tritt neuerdings beschleunigte spontane Athmung auf, und es wiederholt sich der gleiche Vorgang (vergl. Versuch vom 19. 11. 1909).

$\mathrm{Ob}$ es überhaupt gelingt, ein Thier durch Cholin wie durch das Physostigmin 2 ) definitiv $z u$ erhalten, habe ich nicht weiter untersucht.

Die merkwürdige Erscheinung von Seiten der Athmung erklärt sich durch folgenden Versuch: Injicirt man die gleich grosse Dosis (etwa 0,01-0,02) einer ätherisirten Katze, so treten Krämpfe und die gleiche beschleunigte Athmung auf, dor bald eine Athmungslähmung folgt. Wird rechtzeitig künstliche Athmung eingeleitet, so kann Spontanathmung wieder eintreten; ohne arteficielle Respiration geht das Thier zu Grunde.

Eine weitere erwähnenswerthe Erscheinung ist, dass unter dieser wiederbelebenden Wirkung des Cholins beim curaresirten Thiere die Sehnenreflexe wieder auftreten, während der Cornealreflex oft nicht zurückkehrt.

1) Vergl. Pal, Physostigmin, ein Gegengift des Curare. Centralbl. f. Physiol. Wien 1900. August.

2) Vergl. auch Rothberger, Arch. f. Physiol. 1901. 
Das Cholin ist, nach meinen Versuchen zu schliessen, ein Krampfgift, das auch die spinalen Centren reizt, wie aus den Krampfzuständen bei durchtrennter Oblongata, aber erhaltenem Rückenmark hervorgeht. Zu diesem Effect bedarf es aber grösserer Dosen. Kleine Dosen, intravenös injicirt oder auch in die Carotis centralwärts injicirt, erzeugen ganz geringe motorische Effecte. Uebrigens rufen geringe Dosen, in die Carotis injicirt, eine ganz analoge Druckeurve hervor wie bei intravenöser Injection, nur mit längerer Latenz.

Sehr charakteristisch für das Cholin ist jedeufalls auch die Wirkung auf die Thränen- und Speichelsecretion, die sofort nach der Injection eintritt, ebenso bei curaresirten Thieren wie auch nach Zerstörung der Oblongata. Es erzeugt auch reichliche Ausscheidung in die Trachea und Bronchien, die an dem lauten Rasseln (Lungenödem?) kenntlich sind, Erscheinungen, die durch Atropin beseitigt werden.

Nach Modrakowski soll dem richtigen Cholin diese Secretionswirkung fohlen. Das habe ich nicht gefunden.

Hinsichtlich der Darmwirkung kann ich nur Folgendes feststellen: Das Cholin steigert die Darmbewegung nicht. Es verhält sich die Sache hier wie beim Adrenalin. Während der Contraction der BaucheingeweideGefässe sistiren die Pendelbewegungen und erweitert sich der Darm. Wir beobachten hier Erscheinungen, wie ich sie am Darm unter der $\Lambda$ drenalinwirkung seinerzeit beschrieben habe. Ich habe diese Wirkung als den Effect der arteriellen Gefässcontraction aufgefasst und in diesem Sinne auch andere Substanzen zur Begründung dieser Auffassung herangezogen. R. Magnus hat in seinem Versuch am ausgeschnittenen Darm gezeigt, dass speciell bezüglich des Adrenalins oder Suprarenins directe Wirkungen auf Hemmungsnerven in Betracht kommen. Da ich erst vor Kurzem über dieses Thema eine Mittheilung gemacht habe, vorwoise ieh hier auf diese ${ }^{1}$ ).

Nach Ablauf der Gelässcontraction (pressorischen Blutdruckerscheinungen) treten die Pendelbewegungen wieder lebhaft zu Tage. Es ist möglich, dass sich der Darm in diesem Zeitpunkt lebhaft bewegt. Lohmann und ebenso Abderhalden und Fr. Müller fanden an dem nach Magnus hergestellten plexushaltigen Darmpräparat eine Erhöhung des Tonus. Ich habe diesen Versuch bisher nicht wiederholt. Ls steht dies an und für sich nicht in Widerspruch zu meinen Beobachtungen, die sich auf eine ganz andere Versuchsanordnung und auf pressorische Druckwirkungen beziehen. Es ist dies nach meiner Ansicht nicht ein primärer, sondern ein secundärer Effect.

Auch betreffs der Harnausscheidung konnte ich nur in ähnlicher Weise wie am Darm höchstens eine Nachwirkung, keine unmittelbare Wirkung auf die Harnsecretion feststellen.

Ebenso scheint mir die Secretion des Pankreassaftes nicht unmittelbar beeinflusst. Ich habe sie nur einmal geprüft, konnte aber keine directe Wirkung feststellen. Es geht übrigens aus den Protokollen von ModraNo. 39 .

1) Pal, Gefässkrampf und Darmbewegung. Wiener klin. Wochenschr. 1910. 
kowski hervor, dass dic Speichelabsonderung unmittelbar gesteigert wird, während es sich bei der Pankreassecretion um eine Spätwirkung handelt.

\section{Ueber die Wirkung des Neurin.}

Auch hier will ich von der Erörterung der Frage, inwieweit dem Neurin eine physiologische bzw. pathologisch-physiologische Bedentung im Organismus zukommt, absehen. Das synthetisch dargestelle Präparat verdanke ich gleichfalls den Höchster Farbwerken.

Untersuchungen über die Wirkung des Nourins liegen von Formanek, Halliburton und von Lohmann vor.

Nach Formanek ${ }^{1}$ ) wirkt das Neurin auf den Hund (Narkose: Morphin, dann Curare) stark drucksteigernd durch Einwirkung auf die Vasoconstrictoren, und zwar wirkt es sowohl central als auch peripher. Grosse Dosen erniedrigen den Blutdruck wahrscheinlich durch Einwirkung auf das Herz. Injicirt wurde von einer 4 proc. Neurinchloridlösung $1-5 \mathrm{ccm}$.

Aus dem Bericht von W. D. Halliburton (Ergebnisse der Physiologie. 1905. Bd. 4. S. 67) ist zu entnehmen, dass er das Neurin viel giftiger findet als das Cholin, dass es das Herz affieirt, eher eine Constriction als Erweiterung der peripheren Gefässe hervorruft, die Athembewegungen stimulirt und dann lähmt und eine curareähnliche Wirkung auf den willkürlichen Muskel ausübt. Eine abgebildete Curve zeigt eine Depression, die in Drucksteigerung übergeht (Katze, Injection von $2,5 \mathrm{ccm}$ eincr 0,1 proc. Lösung).

Lohmann ${ }^{2}$ ) hat aus der Rinde der Nebenniere eine drucksteigernd wirkende Substanz hergestellt, die er auf Grund chemischer Analyse als Neurin erkannt hat. Die von Lohmann abgebildete Drucksteigerung nach Injection von 0,02 der Substanz ist wohl schr gering.

Es werden sonach dem Neurin fast ausschliesslich vasoconstrictorische Eigenschaften zugeschrieben.

Ich habe 26 Versuche an Katzen ausgeführt. Nchrere von diesen waren mit Cholinversuchen combinirt. Nach meiner Beobachtung besteht manche Aehnlichkeit in der Wirkung zwischen dem Cholin und dem Neurin. Vor Allem schon in der Beziehung, dass die Wirkung des Neurin gleichfalls eine oft ungleiche ist und der Effect von ganz individuellen Momenten nebst den Versuchsbedingungen abhängig ist. Bei subcutaner Anwendung tritt neben Speichel- und Thränenfluss, Erregungserscheinungen eine ausgeprägte Drucksteigerung auf, die später von anhaltenden Drucksehwankungen gefolgt ist. Bei intravenösor Kinbringung wirkt das Neurin in der Regel pressorisch. Dieser drucksteigernden Wirkung geht aber oft eine kurze depressorische Phase voraus, wie bcim Cholin. Pulsverlangsamung, auch Vaguspulse bei durchtrennten Vagis kommen vor ohne die Drucksteigerung hintanzuhalten. Auch hier habe ich nach langer $\Lambda$ cthernarkose, ferner bei einer Curaresorte gesehen, dass dasselbe Neurin, das bei Verwendung eines anderen Curare oder namentlich nach

1) Formanek, Arch. internat. de pharm.-dyn. et de thérapie. 1902. T. 10. p. 273.

2) Lohmann, Arch. f. Physiol. 1909. 
Durchschneidung der Oblongata stark pressorischen Effect hatte, eine muscarinartige Wirkung zeigte, die erst durch grosse Dosen Atropin beseitigt werden konnte.

Bei Verwendung ganz kleiner Dosen ergab sich eine wesentliche Differenz, indem bei einzelnen Thieren die kleinsten Gaben, wie z. B. 0,000001 sehon drucksteigernd wirkten, während bei anderen die gleiche Dosis nur eine ausgeprägte Depression brachte. Ich lege zur Illustration hier einige Curven bei.

Bei manchen, insbesondere nicht narkotisirten Thieren (OblongataDurchtrennung) wirkten 0,00005 wie Adrenin, bei anderen wio Cholin.

Die Wirkung auf den Gefässapparat ist jedenfalls auch hier cine periphere, da auch nach Ausschaltung der vasomotorischen Centren (Durchschneidung der Splanchnici, Zerstörung des Rückenmarks) der pressorische Iffect eintritt.

Hinsichtlich der Herzwirkung gilt auch für das Neurin dasselbe wie für das Cholin.

Das Neurin wirkt auf die motorischen Centren erregend, aber auch auf die peripheren Enden der motorischen Nerven. lis hebt in gleicher Weise wie das Cholin die Curarewirkung vorübergehend auf. Nur sind hierzu kleinere Dosen ausreichend. Meist wirkt 0,001, oft schon cine weit geringere Gabe ganz ausgiebig. Auch hier hebt die Athmungslähmung die Spontanathmung bald auf.

Das Neurin stellt den Dünndarm auf der Höhe seiner pressorischen Wirkung diastolisch still unter Sistirung der Pendelbewegungen, ebenso wie das Adrenalin ${ }^{1}$. Nach Abschluss der Hemmung folgen lebhaftere Bewegungserscheinungen.

Das Neurin erweitert niclit die Pupille des enucleirten Froschauges. Auch auf die Thränen- und Speicheldrüsen wirkt das Neurin wie das Cholin. Eine directe Einwirkung auf die Harnsecretion habe jch auch hier nicht constatiren können.

\section{Zusammenfassumg.}

I.

Das synthetisch dargestellte salzsaure Cholin (Höchst) ist cin haltbares Product und wirkt dennoch ungleich. Es wirkt, intravenös in die Blutbahn gebracht, depressorisch und pressorisch. Nach protrahirter Aethernarkose, nach Verwendung gewisser Curaresorten kann der pressorische Effect ausbleiben. Bei wenig narkotisirten Thieren, wie nach Durchtrennung der Oblongata prädominirt die pressorisehe Blutdruekwirkung.

Das Cholin wirkt auch erregend auf dic Vagusenden im Herzen, jedoch ceteris paribus ganz inconstant, so dass individuelle Reaction des Thieres als maassgebend angenommen werden muss.

Das Cholin wirkt erregend auf die motorischen Centren des Gehirns und des Rückenmarkes und löst clonische Krämpfe aus. Es wirkt auch

1) Pal, Gefässkrampf und Darmbewegung. Wiener med. Wochenschr. 1910. No. 39. 
erregend auf die motorischen Endapparate in den Muskeln und hebt die Curarewirkung auf. Die wiederbelebende Wirkung des Curare ist jedoch nur eine vorübergehende, weil zwar Spontanathmung eintritt, diese aber durch Lähmung des Athmungscentrums aufgehoben wird. Die Lähmung ist bei kleineren Gaben eine vorübergehende, sie kann durch künstliche Athmung überwunden werden.

Das Cholin erregt die Secretion der Thränen- und der Speicheldrüsen durch periphere directe Einwirkung.

Die Wirkung auf die Harnsecretion und wahrscheinlich auch auf die Pankreassecretion ist nur eine indirecte. Ebenso wird der Darm durch die Kreislaufsvorgänge indirect beeinflusst. Die Steigerung der Darmbewegung steht jedenfalls mit diesen in Zusammenhang.

Bei subcutaner Anwendung wirkt dieses haltbare salzsaure Cholin wenig giftig.

II.

Das salzsaure Neurin wirkt (bei Katzen) in vieler Bezichung ganz ähnlich dem Cholin (s. oben), nur ist seine Wirkung (intravenös) cine mächtigere und namentlieh hinsichtlich der Gefässwirkung vorherrschend pressorisch, die mitunter schon in Dosen von unter 0,0001 stark zu Tage tritt. Es hebt die Curarewirkung vorübergehend auf.

Die muscarinartige Wirkung auf das Herz ist eine inconstante und von den Versuchsbedingungen, wie beim Cholin, abhängig.

Schr kleine Gaben machen (intravenös) oft geringe Depression, ohne nachfolgende besonderc Drucksteigerung; bei grösseren ist dic Depression eine kurz vorübergehende.

Subcutan wirkt es drucksteigernd mit nachfolgenden fortlaufenden Schwankungen.

Zur Illustration der angeführten Beobachtungsergebnisse schliesse ich eine Auswahl von Protokollen (zwölf) an.

\section{Versuchsprotokolle.}

Versuch: 19. 11. 1909.

Katze, 1,4 ing. Beginn 6 Uhr 10 Min. abends. Curare (1 Spr.). Vagi durchtrennt. Tief curaresirt. Bei $\Lambda$ thmungsaussetzung keine Krämpfe.

6 Uhr 23 Min. 0,1 Chol. synth. Das Thier zuckt. Blutdr.: $42: 37: 38 \mathrm{~mm}$. -6 Uhr 25 Min. Athmung ausgesetzt - Zuckungen. - 6 Uhr 28 Min. 0,1 Ch. s. Thier unruhig, zeigt Speichelfluss, athmet spontan. Künstliche Athmung ausgesetzt. Druck sinkt vorübergehend ein wenig. Kein Cornealreflex. - 6 Uhr 35 Min. muss wieder künstlich geathmet werden. -- $6 \mathrm{Uhr} 42 \mathrm{Min} .0,2 \mathrm{Ch}$. s. Athmet wieder einige Minuten spontan. Keine Blutaruckwirkung.

Résumé: Nach tiefer Curaresirung Cholin nur von depressorischer Wirkung obwohl Zuckungen auftreten. Wiederholtes Eintreten der Spontanathmung nach der Cholininjection.

$$
\text { Versuch: 25. 11. } 1909 .
$$

Katze, 2,9 kg. Beginn 5 Uhr 45 Min. Curare. Vagi durchtrennt. Tief curaresirt. 5 Uhr 0,2 Ch. s. intravenös. Das Thier beisst während der Einspritzung $(2 \mathrm{~cm})$, Krämpfe, athmet spontan. Cornealreflex nicht nachweisbar. Starker Speichelfluss, Trachealrasseln. Blutdruck $72: 60: 86$, vorübergehend Andeutung von Vaguspulsen. 
Athmung ausgesetzt, da die spontane Athmung genügt. - 5 Uhr 21 Min. 0,005 Atropin. - 5 Uhr 32 Min. 0,1 Ch. s. intravenös. Blutdruck: $70: 65: 79: 75$, dann ziemlich steiler Anstieg auf $176 \mathrm{~mm}$. Athmet ausgiebig spontan. - 5 Uhr 35 Min. Athmung seltener. - 5 Uhr 37 Min. Spontane Athmung hat aufgehört. - 5 Uhr $38 \mathrm{Min} .0,1 \mathrm{Ch}$. s. Geringe träge Blutdruckwirkung $(32: 40: 24: 46 \mathrm{~mm})$. Athmet eine kurze Zeit lang wieder spontan, dann Exitus.

Résumé: Tief curaresirtes Thier erwacht noch während der Injection mit Krämpfen, trotzdem kaum merklicher Anstieg. Einige Zeit anhaltende Spontanathmung. Nach Atropin die initiale Depression nicht ganz geschwunden. Starke Drucksteigerung. Nach einer weiteren gleichstarken Dosis Cholin ausgesprochene Depression.

Versuch: 11. 1. 1910.

Katze, 3,3 kg. Beginn des Versuches 5 Uhr 50 Min. Medulla oblongata durchtrennt. Ballonschreibung aus dem Dünndarm.

6 Uhr 45 Min. 0,1 Ch. s. intravenös. Blutdruck $62: 56: 42$, rascher Anstieg a uf $212 \mathrm{~mm}$. Auf der Höhe des Blutdruckes sistiren die Pendelwellen des Darmes, der Darm wird weiter. Thränenfluss, Speichelfluss, Lungenödem. -- 6 Uhr 53 Min. leichte Krämpfe in den Extremitäten. - $6 \mathrm{Uhr} 57 \mathrm{Min} .0,05 \mathrm{Ch}$. s. intravenös. Blutdruck $41: 43: 36$, steiler Anstieg a uf $198 \mathrm{~mm}$. Darmwellen wie bei der vorigen Injection. -7 Uhr $10 \mathrm{Min}, 0,004$ Morphin. hydrochlor. Pendelwellen werden unter mässiger Contraction des Darmes wesentlich grösser. - 7 Uhr 13 Min. 0,025 Ch. s. Blutdruck $37: 45: 40$, rascher Anstieg auf $144 \mathrm{~mm}$. Während des hohen Druckes sistiren die Pendelwellen unter Erweiterung des Darmlumens. - 7 Uhr 17 Min. 0,01 Ch. s. Blutdruck $34: 70 \mathrm{~mm}$. Während der Drucksteigerung versiegen die Darmwellen.

Obduction: Medulla oblongata zerstört.

Résumé: Nach Durchtrennung der Medulla oblongata wirkt Cholin lebhaft drucksteigernd. Krampferscheinungen folgen erst. Während der Drucksteigerung ruhen die Pendelwellen des Dünndarmes.

Versuch: 25. 1. 1910.

Katze, $2,7 \mathrm{~kg}$. Medulla oblongata durchtrennt. Vagi durchschnitten. Künstliche Athmung. Beginn des Versuches 5 Uhr 50 Min.

6 Uhr 4 Min. 0,2 Ch. s. subcutan (=2,0 der 10 proc. Lösung) in die 'Thoraxhaut injicirt. - 6 Uhr 10 Min. leichte Zuckungen in den Hinterbeinen. - 6 Uhr 13 Min. 'Zuckungen in den oberen Extremitäten. - 6 Uhr 17 Min. Krampfanfall in allen Extremitäten. - 6 Uhr 28 Min. Krämpfe mit Druckanstieg von $76: 100 \mathrm{~mm}$, kein Speichelfluss bemerkbar. -6 Uhr 30 Min. 0,008 Ch. s. intravenös. -6 Uhr $30^{1} / 2$ Min. allgemeine Krämpfe mit nachfolgendem fibrillärem Zucken. Thränen- und Speichelfluss. Blutdruck $76: 56: 87 \mathrm{~mm}$. $-6 \mathrm{Uhr} 35 \mathrm{Min} .0,02 \mathrm{Ch}$. s. intravenös. Sofort allgemeine Krämpfe. Blutdruck $64: 74: 58: 212 \mathrm{~mm}$. Thränen- und Speichelfluss zugenommen. $-6 \mathrm{Uhr} 39-40 \mathrm{Min}$. Athmung ausgesetzt, Krämpfe getinger, doch anhaltend. - 6 Uhr $42 \mathrm{Min} .0,02 \mathrm{Ch}$. s. intravenös. Blutdr. $66: 82: 66: 200 \mathrm{~mm}$. Krämpfe gesteigert. - 6 Uhr 51 Min. 0,002 Atropin. - 6 Uhr 53 Min. 0,02 Ch. s. intravenös. Vorübergehend Zuekungen. Blutdruek $56: 62: 40: 112 \mathrm{~mm}$. - $6 \mathrm{Uhr}$ 57 Min. 0,002 Atropin. - 6 Uhr 59 Min. 0,05 Ch. s. intravenös. Zuckungen. Blutdruck $41: 51: 68: 88 \mathrm{~mm}$. -7 Uhr 2 Min. Athmung ausgesetzt.

Obduction: Medulla oblongata, auch das oberste Halsmark zerstört.

Résumé: Dieser Versuch zeigt die Wirkung der subcutanen Injection bei durchtrennter Oblongata, dann starke Drucksteigerung bei intravenöser lnjection. Nach Atropin steigert sich die Wirkung nicht. 
Katze, $3,7 \mathrm{~kg}$. Beginn 5 Uhr $40 \mathrm{Min}$. Medulla oblongata durchtrennt. Vagi durchschnitten. Zuckungen im rechten Bein.

5 Uhr 53 Min. 0,01 Cholin hydrochl. intravenös. Blutdruck $90: 80: 140 \mathrm{~mm}$, geringe Krämpfe, Speichel- und Thränenfluss. - $6 \mathrm{Uhr}$ beide Splanchnici im T'horax durchtrennt. - 6 Ubr 10 Min. 0,03 Ch. hydrochl. intravenös. Blutdruck $33: 31: 236 \mathrm{~mm}$. Zuckung im r. Bein. - 6 Uhr 16 Min. 0,03 Cholin. hydrochl. intravenös. Blutdruck $56: 59: 55: 212 \mathrm{~mm}$. -6 Uhr 24 Min. 0,03 Cholin. hydrochl. intravenös. Blutdruck $54: 56: 52: 188 \mathrm{~mm}$. - 6 Uhr 34 Min. 0,4 Arterenol $1 \mathrm{pm}$. Blutdruck $52: 252 \mathrm{~mm}$.

Obduction: Oblongata bis auf einen seitlichen Faden durchtrennt.

Résumé: Starke, pressorische Wirkung mit minimalster Depression nach Durchtrennung der Oblongata und beider Splanchnici.

\section{Versuch: 8. 2. 1910.}

Katze, $2,5 \mathrm{~kg}$. Beginn 6 Uhr 15 Min. abends. Curare $(3 / 4 \mathrm{Spr}$.). Vagi durchtrennt. In die linke Carotis cardialwärts zur Registrirung, cerebralwärts zur Injection eingebunden.

6 Uhr 32 Min. 0,0075 Ch. s. in die 1. Carotis cerebralwärts, Thränen- und Speichelsecretion. Blutdr. $97: 88$, allmählich aufsteigend bis 96 . Keine Krämpfe. 6 Uhr 36 Min. physiol. Kochsalzlösung in die Carotis nachgespritzt $(1,0)$. Blutdruek $96: 82: 94 .-6$ Uhr 39 Min. 0,0075 Ch. s. intravenös. Blutdr. $96: 102: 78: 102$, sehr träger Anstieg. - 6 Uhr $42 \mathrm{Min}$ 0,05 Ch. s. intravenös. Das Thier beisst schon während der Einspritzung, hat bald Cornealrellex, athmet sofort spontan, hat reichlichen 'Thrïnen- und Speichelfluss. Blutdruck 96:102: 74:112. Athmung ausgesetzt, athmet spontan. -6 Uhr 50 Min. $0,05 \mathrm{Ch}$. s. in die 1. Carotis centralwärts injicirt. Pupille wird enger. Blutdruck $96: 100: 80: 93 \mathrm{~mm}$. - 6 Uhr 55 Min. 0,1 Ch.s. intravenös. Athmet stärker, bewegt sich lebbaft. Blutar. 80:90:76:102 mm. -- 6 Uhr 58 Min. Athmet ausgiebig. Cornealreflex lebhaft. Blase niüssig gefüllt. 7 Uhr 0,04 Ch. s. in die linke Carotis. Pupille wird enger. Schnappt bei Berührung der Lippen. Reagirt auf Kneifen. $\Lambda$ uf Blutdrack ohne Einfluss. - 7 Uhr 5 Min. 0,1 Ch. s. intravenös. Keine Druclisteigerung. Das Thier will sich losreissen. Sehr reichlicher Speichel- und Thränenfluss, auch Nasenschleim wird sichtbar. - 7 Uhr 8 Min. 0,1 Ch. s. in die Carotis. Schweifliämpfe, hat Reflexe. - 7 Uhr 14 Min. $1 / 2$ Spritze Curare. - 7 Uhr 19 Min. Beide Splanchnici im Thorax durchtrennt. 0,1 Ch. s. intravenös. Kein Einfluss auf den Blutdruck. Das Thier reagirt wieder. 7 Uhr 25 Min. 0,1 1 prom. Arterenol. Blutdruck $42: 180 \mathrm{~mm}$.

Résumé: Der Versuch illustrirt die Wirkung am curaresirten Thier bei Injection in die Carotis (cerebral) und-intravenöser Anwendung.

\section{Versuch: 16. 2. 1910.}

Katze, $2,2 \mathrm{~kg}$. Beginn $5 \mathrm{Uhr} 35 \mathrm{Min}$. Aethernarliose. Medulla oblongata durchtrennt. Rückenmark ausgebohrt, davon $22 \mathrm{~cm}$ entfernt.

6 Uhr $10 \mathrm{Min}$. Beginn der Blutdruckschreibung. Leichte Zuckung in der rechten Pfote. - 6 Uhr 11 Min. 0,05 Ch. s. intravenös injicirt. Reichlicher Thränen- und Speichelfluss. Blutdruck $44: 47: 43 \mathrm{~mm}$, dann rascher Anstieg auf $136 \mathrm{~mm}$. 6 Uhr $16 \mathrm{Min}$. $0,02 \mathrm{Ch}$ s. intravenös. Blutdruck $46: 70 \mathrm{~mm}$. - 6 Uhr $20 \mathrm{Min}$. 0,1 Ch. s. intravenös. Blutdruck $48: 50: 48: 114 \mathrm{~mm}$, rascher Anstieg. - $6 \mathrm{Uhr}$ 25 Min. 0,2 der 1 proc. Artorenollösung. Blutdruck $46: 118 \mathrm{~mm}$ - - 6 Uhr $30 \mathrm{Min}$. 0,5 derselben Lösung. Blutdruck $52: 184 \mathrm{~mm}$. -- 6 Uhr 34 Min. 0,1 Ch. s. Blutdruck $52: 56: 51: 63 \mathrm{~mm}$.

Obduction: Medula oblongata durchtrennt. Halsmark zerquetscht. Das Brustund Lendenmark entfernt. 
Versuch: 17. 2. 1910.

Katze, 3,0 kg. Beginn 5 Uhr 45 Min. Medulla oblongata durchtrennt. Thorax eröfnet. Herz im Rothberger'schen Plethysmographen.

6 Uhr 13 Min. 0,05 Cb.s. intravenös. Speichel- und Thränenfluss. Vaguspulse - Herzcurve entsprechend - Schlagvolumen erhöht. Blutdruck $58: 64: 50: 198 \mathrm{~mm}$. - 6 Uhr 16 Min. Atropin 0,002. - 6 Uhr 18 Min. 0,05 Ch. s. Blutdruck $64: 67: 62: 138 \mathrm{~mm}$. - 6 Uhr $22 \mathrm{Min} .0,1 \mathrm{Ch}$. s. Blutdruck $50: 168 \mathrm{~mm}$. - $6 \mathrm{Uhr}$ 25 Min. Curare ( $3 / 4$ Spritze). - 6 Uhr 30 Min. 0,1 Ch. s. Blatdrack $64: 34 \mathrm{~mm}$. 6 Uhr 33 Min. 0,2 Arterenol. Blutdruck $46: 114 \mathrm{~mm}$. - 6 Uhr 37 Min. 0,25 Arterenol. Blutdruck $50: 150 \mathrm{~mm}$. - 6 Uhr $44 \mathrm{Min}$. 0,1 Ch. s. ohne Wirkung auf den Blutdruck. Erstickung ohne Krämpfe.

Obductionsbefund: Medulla oblongata vollkommen durchtrennt, theilweise zerstört.

Diese Katze wurde drei Wochen vorher aus der Aethernarkose durch Physostigmin wieder belebt.

Résumé: Nach Durchtrennung der Oblongata steigert Atropin nicht den Effect. Curareinjection hebt die pressorische Wirkung auf.

\section{Versuch 17. 5. 1910.}

Beginn 5 Uhr $30 \mathrm{Min}$. Katze, $2,5 \mathrm{~kg}$. Aethernarkose. Vagi durchtrennt.

5 Uhr 50 Min, 0,0025 Cholin. hydrochl. intravenös. Blutdr. 144:128:140 mm. -5 Uhr 56 Min. 0,005 Ch. h. $128: 106: 127 \mathrm{~mm}$. - 6 Uhr Curare $(0,4)$. - 6 Uhr 6 Min. 0,01 Ch. h. Blutdruck $40: 44: 43: 55 \mathrm{~mm}$.

Résumé: In anhaltender Aethernarkose wirkte hier das Cholin depressorisch.

\section{Vorsuch: 6, 6. 1910 .}

Katze, $3,5 \mathrm{~kg}$. Beginn 5 Uhr $45 \mathrm{Min}$. Tiefe Aethernarkose bis Athmungsstillstand. Vagi intact. Blase voll, nicht ausdrückbar.

6 Uhr 3 Min. Curare. - 6 Uhr 5 Min. 0,01 Neurin. hydrochl. Blatdr. 118:128 mm. Spontane Blasenentleerung. - 6 Uhr 9 Min. 1 Spritze Curare. -- 6 Uhr 14 Min. 0,01 Neurin. hydrochl. Blutdruck 100:214 mm. - 6 Uhr 19 Min. 0,005 Neurin. hydrochl. Blutdruck $88: 86: 176 \mathrm{~mm}$.- $6 \mathrm{Uhr} 23 \mathrm{Min}$. 0,003 Cholin hydrochl. Blutdruck $84: 74: 78 \mathrm{~mm}$. - 6 Uhr $27 \mathrm{Min}$. 0,01 Cholin. hydrochl. Blutdruck $72: 66:: 78 \mathrm{~mm}$ (ganz allmählicher Anstieg). -- 6 Uhr 30 Min. 0,005 Atropin. 6 Uhr 32 Min. 0,01 Cholin. hydrochl. Blutdruck 108:112:108:122 mm, später Zuckungen. Das 'Thier hat Rellexe. - 6 Uhr 361/2 Min. 0,01 Neurin. hydrochl.; steiler Druckanstieg von $92: 258 \mathrm{~mm}$. Das Tier macht Athmungsbewegungen mit dem Mund, athmet jedoch nicht spontan. Das Zwerchfell steht. - 6 Uhr 48 Min. 0,01 N. hydrochl. Blutdruck $70: 252 \mathrm{~mm}$, wie oben.

Résumé: Dieser Versuch zeigt die Neurinwirkung, die auch nach Curare eine pressorische ist, während Cholin depressorisch wirkt und auch nach Atropin nur geringe Drucksteigerung hervorruft.

\section{Versuch: 15.7 .1910$.}

Katze, $3 \mathrm{~kg}$. Beginn 6 Uhr 5 Min. Leicht curaresirt ( $1 / 2$ Spritze). Vagi durchtrennt.

6 Uhr 33 Min. Curare $(0,3)$ nachinjicirt. - 6 Uhr 35 Min. 0,1 Neurin. hydrochl. in 1,0 Kochsalzlösung subcutan. Blutdruck $110 \mathrm{~nm},-6$ Uhr $40 \mathrm{Min}$ auf $176 \mathrm{~mm}$ ganz allmählich angestiegen, - 6 Uhr 49 Min. $58 \mathrm{~mm}$. - 6 Uhr 50 Min. 0,0012 N. hydrochl. intravenös. Blutdruck $58: 136 \mathrm{~mm}$. - 6 Uhr 55 Min. 0,0006 Neurin. hydrochl. Blutdruck $86: 81: 128 \mathrm{~mm}$. 


\section{Versuch: 19. 7. 1910.}

Katze, $3,5 \mathrm{~kg}$. Beginn 5 Uhr 40 Min. Vagi durchtrenut. Neue Curarelösung.

6 Uhr 2 Min. 0,000001 Neurin. hydrochl. intravenös ohne Effect. - 6 Uhr 5 Min. 0,000001 Neurin hydrochl. Blutdr. 100:102 mm. - 6 Uhr 7 Min. 0,000001 Neurin hydrochl. Blatdruek $98: 102 \mathrm{~mm}$. - $6 \mathrm{Ubr} 12 \mathrm{Min}, 0,000005$ Neurin. hydrochi. Blutdruck $96: 931 / 2: 97 \mathrm{~mm}$. -6 Uhr 14 Min. 0,00001 Neurin. hydrochl. Blutdruck $92: 96 \mathrm{~mm}$. -6 Uhr 17 Min. 0,00001 Neurin. hydrochl. Blutdruck $92: 88: 98 \mathrm{~mm}$. Thier reagirt. - 6 Uhr 20 Min. 0,00005 Neurin. hydrochl. Blutdruck $90: 98 \mathrm{~mm}$. Thier wach. - 6 Uhr 23 Min. 0,0001 Neurin. hydrochl. Blutdruck 98:116 mm. 'Thier wach, athmet spontan.

Résumé: Der Versuch zeigt die Aufhebung der Curarewirkung durch minimale Mengen von Neurin. 


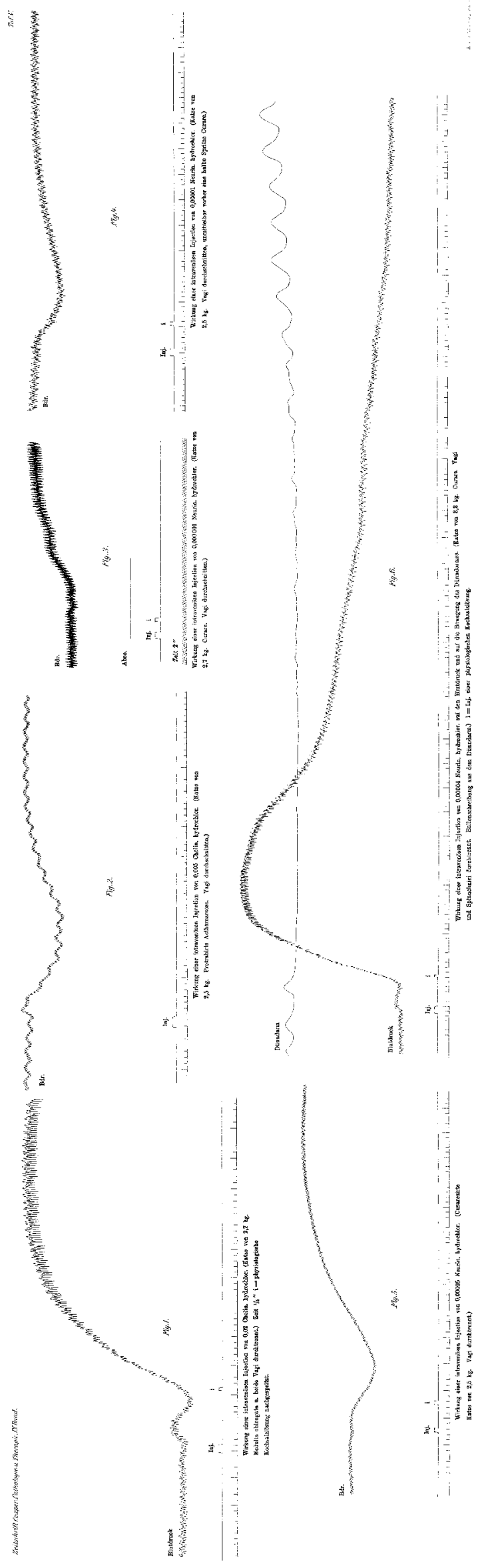

